Le Parlement examinera prochainement la nouvelle loi sur les produits du tabac censée protéger la population contre les effets nocifs du tabagisme. Suite au message du Conseil fédéral, la question se pose de savoir ce qui prime réellement: la protection de la santé de la population ou les intérêts économiques de l'industrie cigarettière. Lors de la consultation, la FMH a déjà souligné sa principale critique dans une prise de position largement soutenue par différentes organisations médicales (cf. page 1750 du présent numéro du BMS): la publicité sur le tabac doit être exclue de tous les espaces de vie, et en particulier de ceux des enfants et des adolescents.

\title{
Tout n'est donc que bruit et fumée?
}

\section{Barbara Weil}

Cheffe de la division Promotion de la santé et prévention

Dans le monde entier, et aussi en Suisse, l'efficacité de la prévention contre le tabac suscite le débat depuis des années.

Le fait est que 6 millions de personnes meurent chaque année du tabagisme dans le monde, et près de 9000 en Suisse. De plus, les décès prématurés liés à la consommation de tabac engendrent des coûts annuels directs et indirects à hauteur de 10 milliards. L'OMS classe la fumée parmi les facteurs à risque des maladies très répandues comme le cancer, les maladies cardiaques, les affections pulmonaires et le diabète. Le fait est que la publicité pour le tabac a avant tout un impact sur les jeunes, et donc sur leur entrée dans le monde des fumeurs. L'argument du libre arbitre n'a aucun sens tant que la publicité pour les produits du tabac sera omniprésente et à la portée des enfants et des adolescents, que ce soit sur les présentoirs, entre chewing-gums et bonbons, ou lors de festivals en plein air, où les jeunes ont la possibilité de tester de nouvelles cigarettes ou de faire le plein de gadgets offerts par l'industrie du tabac.

\section{Personne n'est à l'abri de la publicité pour le tabac, encore moins les jeunes et les adolescents.}

Le fait est que plusieurs multinationales du tabac ont leur siège principal en Suisse. Non seulement elles offrent plus de 4000 emplois mais en plus elles constituent une source de revenus fiscaux importante pour les cantons. Et accessoirement, dans un esprit scientifique, elles financent la recherche sur le tabac ou soutiennent des études qui remettent en question la soidisant efficacité des paquets neutres, en arguant que ces derniers enflamment uniquement la guerre des prix, la contrebande et la contrefaçon sans inciter à cesser de fumer. Elles contestent simplement l'évidence scientifique de la prévention et de la médecine. Le fait est aussi que la Convention-cadre de l'OMS pour la lutte antitabac a été signée par 168 pays en 2003. Si certains Etats, comme l'Australie, l'Irlande ou l'Angleterre, vont plus loin avec notamment l'idée des paquets neutres, qui empêchent aux cigarettiers de distinguer leur marque par rapport à la concurrence, ils doivent faire face à des plaintes devant des juges internationaux, orchestrées par le lobby du tabac, lorsqu'ils introduisent les mesures légales nécessaires.

\section{Seule protection efficace: interdire strictement} la promotion, le parrainage et la publicité en faveur du tabac et des cigarettes électroniques.

Le fait est enfin, et c'est le plus intéressant du point de vue de la prévention, que la jeunesse australienne n'a jamais trouvé la cigarette aussi peu séduisante qu'aujourd'hui. «Le paquet standardisé n'agit plus comme un symbole d'appartenance à un groupe particulier», déclare Simon Chapman, le père spirituel du paquet neutre australien. Dans les magasins et autres points de vente, les cigarettes ne sont plus accessibles librement. Le vendeur ne sort le paquet souhaité qu'une fois qu'un client en fait activement la demande. Aujourd'hui, seuls $12,8 \%$ de tous les plus de 14 ans fument en Australie, alors que la Suisse en dénombre encore le double.

Qu'est-ce que tout cela signifie pour la prévention et la protection de la santé des jeunes et des adolescents de notre pays? Le corps médical exige toujours une prévention efficace contre le tabagisme et une interdiction globale et stricte de la promotion, du parrainage et de la publicité pour les produits du tabac et les cigarettes électroniques. Si, pour protéger les enfants et les adolescents, on veut réduire les dommages découlant du tabagisme et diminuer la perte de qualité de vie, la publicité en faveur du tabac doit disparaître de tous les espaces de vie.

La FMH espère que nos politiciens se prononceront pour une protection efficace et que les efforts menés jusqu'à présent par la prévention ne se perdront pas dans un écran de fumée... 